\title{
A Novel Surface Texture Shape for Directional Friction Control
}

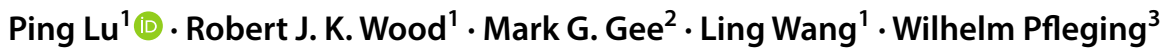

Received: 7 November 2017 / Accepted: 29 January 2018 / Published online: 14 February 2018

(c) The Author(s) 2018. This article is an open access publication

\begin{abstract}
An experimental study is presented to evaluate the influence of anisotropically shaped textures on the behaviour of sliding friction and sensitivity to sliding direction. The plate samples were textured with triangular sloped dimples using an ultrafast laser surface texturing technique. Reciprocating cylinder-on-plate tests were conducted with steel sliding pairs using mineral base oil as a lubricant to compare the tribological performance of reference non-textured specimen and dimpled samples. The dimples were designed with varying converging angles in the transverse $y-z$ plane and top-view $x-y$ plane. In this study, no dimple was fully covered in the contact area since the dimples size is much larger than the Hertzian line contact width. Stribeck style dynamic friction curves across boundary, mixed and hydrodynamic lubrication regimes were used to determine the benefit or antagonism of texturing. Observation of the directional friction effect of the anisotropic textures indicated that the converging shapes are beneficial for friction reduction, and the dimpled specimens have a lower friction coefficient particular under prevailing boundary lubrication conditions. It was also found that the real contact length variation rate is a major factor controlling the local friction response. The sloped bottoms of the textures produce effective converging wedge action to generate hydrodynamic pressure and contribute to the overall directional friction effects.
\end{abstract}

Keywords Anisotropically surface texturing $\cdot$ Directional effect $\cdot$ Ultrafast laser surface texturing $\cdot$ Reciprocating sliding

\section{Introduction and Background}

Due to long-term evolution and development, many biological surfaces (including both plants and animals) have unique functions to be energy efficient; such as drag reduction (shark's skin) [1], wear resistance (beetle's body) [2], super

Ping Lu

lupingping719@gmail.com

Robert J. K. Wood

r.wood@soton.ac.uk

Mark G. Gee

mark.gee@npl.co.uk

Ling Wang

ling.wang@soton.ac.uk

Wilhelm Pfleging

wilhelm.pfleging@kit.edu

1 National Centre for Advanced Tribology at Southampton, Southampton SO17 1BJ, UK

2 National Physical Laboratory, Teddington TW11 0LW, UK

3 Karlsruhe Nano Micro Facility,

Hermann-von-Helmholtz-Platz 1,

76344 Eggenstein-Leopoldshafen, Germany hydrophobicity (lotus' surface) [3,4] and smart adhesives (gecko's foot) [5]. Surface topography is one of the most important factors for achieving this performance. Inspired by the multi-functional surface of animals and plants, researchers are producing artificial surfaces with regular asperities to determine the shape, size and distribution to obtain the desired surface and tribological properties. This area of research is called surface texturing. For tribologists, one of the main concerns is how to use textured surfaces to control the frictional behaviour under various conditions, either to increase or to decrease friction depending on the requirements of a potential application, such as engine cylinder liner to piston ring contacts.

Since 1965 , more than 400 publications on surface texturing can be found, and the majority of these have been conducted in the past 2 decades [6]. Among them, more than half of the studies are purely theoretical, based on different forms of the Reynolds, Navier-Stokes or Stokes equations. Basic models for various applications, like journal bearings [7], mechanical seals [8], thrust bearings [9] and cylinder liners [10], have been built to find the optimal texture designs. But factors such as oil viscosity, density and temperature, as well as surface deformation were 
neglected in the early models. With the development of more efficient algorithms and solution techniques, models capable of describing viscous heat dissipation and nonNewtonian lubricant behaviour were developed [7].

In the other all publications, around one-third of them involve experimental approaches. Most experiments were conducted with traditional tribometers (unidirectional pinon-disc [11-16] or reciprocating sliding [17-24] tests), while other studies were conducted on real components, including journal bearings [25] thrust bearings [26-28] and mechanical seals [29-31]. To date, various surface texturing techniques have already been introduced, such as laser surface texturing (LST); reactive ion etching (RIE); embossing technique; "Lithographie, Galvanoformung, Abformung" (LIGA) process; abrasive jet machining (AJM), electrolytic etching, and so on. The development of advanced manufacturing techniques allows designers to obtain the desired structures accurately, which makes it possible to validate the surface frictional properties caused by dimple structures experimentally.

As has been discussed in papers published by current authors [32, 33], dimples parameters (dimple depth, dimple diameter, depth-to-diameter ratio, and the texture density, dimple profiles (flat, spherical and linearly varying bottoms) and dimple shapes (circular, elliptical, rectangular, triangular and chevron shapes) have been studied in the past decades. Researchers have been aware of the benefit of anisotropically textured surfaces through numerical simulation, that the converging shape in the $x-y$ plane (triangular dimples) and in the $x-z$ plane (sloped bottom dimples) could both be reasons for enhancing the lubrication effects $[34,35]$. It has also been proved by current authors that anisotropic designed dimples on the surface will make the surface have directional friction properties.

The current research is a follow-up of a recently published work [32, 33], aims at gaining a better understanding of the mechanisms that friction reduction effects and directional effects produced by dimpled surfaces at reciprocating speeds of the lubricated line contacts. To show whether these factors cause a significant impact, three samples with novel textured designs (triangular top-view shapes and wedge bottom shapes) were created by ultrafast laser surface texturing technique. The directional friction study of anisotropic surface texturing could not only enhance the mechanistic understanding of how textured surface works under lubricated conditions, but also broaden the application area of surface texturing, where differential friction induced by sliding directions is required. The results from this work also serve as a basis for using anisotropic surface textures for developing models aiming to decrease friction in lubricated sliding contacts. It records average friction as well as per dimple friction over a range of lubrication regimes.

\section{Method and Material}

The tribological behaviour of ultrafast laser textured and smooth reference surfaces were tested using a cylindricalon-flat line contact configuration with a Plint TE77 reciprocating tribometer (Phoenix Tribology Ltd., Kingsclere, UK). Each test was completed with a new roller on a new track of the plate surface in pure sliding motion.

ASP 2023 plates and bearing steel AISI 52100 rollers were chosen to test the dimple effect through various lubrication conditions. The AISI 52100 cylindrical rollers were chosen to test the dimple effect through various lubrication conditions. The AISI 52100 cylindrical rollers were provided by Bearingboys Ltd. (Bearingboys Ltd., Norfolk, UK) with a dimension of $\emptyset 6 \mathrm{~mm} \times 10 \mathrm{~mm}$ and the hardness of them is $848 \mathrm{HV}$. Both ends of the cylindrical roller had a fillet to avoid the stress concentration. The rollers had an average surface roughness of $R_{\mathrm{q}}=0.03 \mu \mathrm{m}$. An ASP 2023 steel plate provided by ERASTEEL (Eramet Alloys UK Ltd., Sheffield, UK) was cut into TE77 plate test samples with a dimension of $22 \mathrm{~mm} \times 60 \mathrm{~mm} \times 4 \mathrm{~mm}$ before they were sent to Tamworth Heat Treatment (Tamworth Heat Treatment Ltd., Tamworth, UK) for heat treatment to increase the hardness to $800 \mathrm{HV}$. The testing surface of the plates was ground and lapped with different grades of diamond paste using a Kemet lapping machine (Kemet International Ltd., Maidstone, UK) to a surface roughness around $R_{\mathrm{q}}=0.01 \mu \mathrm{m}$ before texturing.

The triangular sloped textures on the steel substrate surfaces were fabricated by the Karlsruhe Nano Micro Facility using laser surface texturing (LST) technique. Ultrafast laser structuring was carried out on a micro-machining workstation (PS450-TO, Optec s.a., Belgium) equipped with a tunable fibre laser (Tangerine, Amplitude Systèmes, France). The average power was $20 \mathrm{~W}$, and the maximum pulse energy was specified to $100 \mu \mathrm{J}$ at $1030 \mathrm{~nm}$ (TEM00 with $M^{2}<1.3$ ). The pulse repetition rate could be varied from single pulse to $2 \mathrm{MHz}$ and the laser pulse duration from 350 to $10 \mathrm{ps}$, respectively. The laser beam was scanned over the sample surface for all experiments using a RhothorTM Laser Deflection Systems scan head (Newson Engineering BV). The laser source can operate at different wavelengths such as in the near infrared $(1030 \mathrm{~nm})$, visible $(515 \mathrm{~nm})$ and UV $(343 \mathrm{~nm})$ region. In this work, three triangular sloped textured samples (TS1, TS2 and TS3) were prepared, the geometry parameters are defined in Fig. 1, and the details of those three samples are found in Table 1. The sloped bottom shape provides a covering/diverging shape in $x-z$ plane, and the top-view triangular shape presents a converging/ diverging shape in the $x-y$ plane. The top-view angle $(\alpha)$ was varied from $14^{\circ}$ to $53^{\circ}$, and slope angle $(\beta)$ was varied from $1.7^{\circ}$ to $6.8^{\circ}$, while the dimple density was fixed at $10 \%$. 


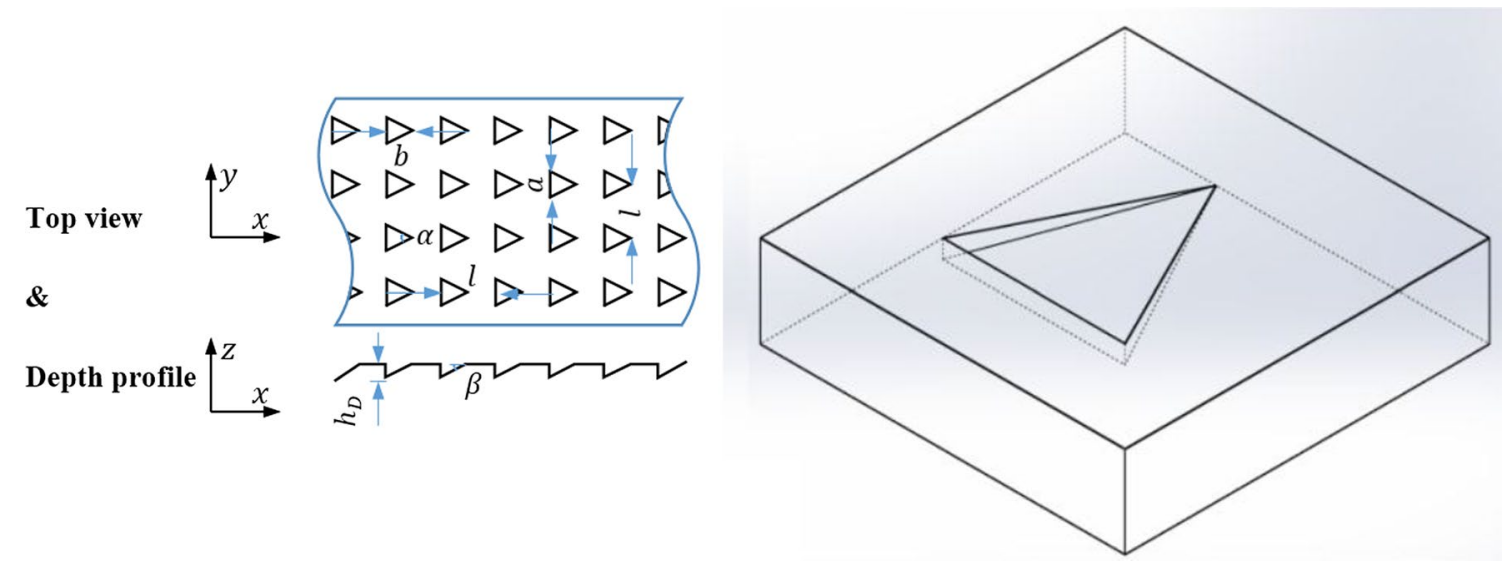

Fig. 1 Parameter symbols in design

Table 1 Parameters of triangular sloped (TS) bottom dimple designs

\begin{tabular}{lllllllc}
\hline Sample No. & $\begin{array}{l}\text { Side } \\
\text { length }(a / \\
\mu \mathrm{m})\end{array}$ & $\begin{array}{l}\text { Height } \\
\text { length }(b / \\
\mu \mathrm{m})\end{array}$ & $\begin{array}{l}\text { Dimple } \\
\text { depth }\left(h_{\mathrm{D}} /\right. \\
\mu \mathrm{m})\end{array}$ & $\begin{array}{l}\text { Top-view } \\
\text { angle }\left(\alpha /^{\circ}\right)\end{array}$ & $\begin{array}{l}\text { Slope } \\
\text { angle } \\
\left(\beta /^{\circ}\right)\end{array}$ & $\begin{array}{l}\text { Textur- } \\
\text { ing density } \\
\left(\rho_{\text {texture }}\right)\end{array}$ & $\begin{array}{l}\text { Interval } \\
\text { length }(l / \\
\mu \mathrm{m})\end{array}$ \\
\hline TS1 & 125 & 500 & 15 & 14 & 1.7 & 10 & 560 \\
TS2 & 250 & 500 & 30 & 28 & 3.4 & 10 & 790 \\
TS3 & 500 & 500 & 60 & 53 & 6.8 & 10 & 1118 \\
\hline
\end{tabular}

A mineral base oil was used in this study to minimise the influence of other factors to focus the study of the influence of textures on friction. For each test, around $15 \mathrm{ml}$ Vitrea 32 mineral base oil from Shell (Shell U.K. Limited, London, UK) was used to ensure that the plate specimen was submerged. The oil temperature was maintained at $24^{\circ} \mathrm{C}$, and the temperature of the oil was stabilised at $32 \pm 1{ }^{\circ} \mathrm{C}$ after running in, and the oil is heated from the frictional heating rather than from the heater bath. For the selected lubricant under this temperature, it has the following properties: $\alpha=12.8 \times 10^{-9} \mathrm{~m}^{2} / \mathrm{N}$, kinematic viscosity $48 \mathrm{~mm}^{2} / \mathrm{s}$, and the dynamic viscosity $\eta_{0}=0.042 \mathrm{~Pa}$ s. The specific film thickness, known as $\lambda$ ratio, was used to define the lubrication regime and the minimum mid-stroke film thickness was calculated using Dowson and Higginson equations for line contacts. Additionally, the dimensionless parameter $\eta V L / W$ was used to produce Stribeck curves, where $\eta$ is the lubricant dynamic viscosity, $V$ is the contact speed, $L$ is the roller length, and $W$ is the normal load. The loading conditions were set to be 100 and $350 \mathrm{~N}$, with the mid-stroke sliding speed ranging from 0.03 to $0.47 \mathrm{~m} / \mathrm{s}$. Under these test conditions, the mid-stroke $\lambda$ ratio ranges from 0.5 to 3.7 , while the Stribeck parameter ranges from $3.77 \times 10^{8}$ to $1.98 \times 10^{6}$.

For each test, the plate specimen was fully submerged by $15 \mathrm{ml}$ of lubricating oil, which were verified by visual inspection. The lubricated friction tests were started at a constant normal load of $100 \mathrm{~N}$, while the reciprocating frequency was changed from 15 to $1 \mathrm{~Hz}$ in a stepwise manner ( $1 \mathrm{~Hz}$ step). The stroke length was set to $10 \mathrm{~mm}$. The test duration at each frequency was set to $8 \mathrm{~min}$, except $18 \mathrm{~min}$ for the $15 \mathrm{~Hz}$ condition (including $10 \mathrm{~min}$ running in period). In the second step, the normal load was increased to $350 \mathrm{~N}$ while the frequency was also changed from 15 to $1 \mathrm{~Hz}$. The initial Hertzian line contacts pressure and contact width of the contact area under certain loads were calculated by the cylinder on plane equations. The $100 \mathrm{~N}$ normal load resulting in a Hertzian mean contact pressure of $0.28 \mathrm{GPa}$, with the initial Hertzian contact width at $36 \mu \mathrm{m}$. While with the $350 \mathrm{~N}$ normal load, the Hertzian mean contact pressure and initial contact width increased to $0.52 \mathrm{GPa}$ and $68 \mu \mathrm{m}$, respectively. However, it should also be noted that the Hertzian contact pressure varied with the real contact length, that contact pressures were higher when the roller was sliding above the dimple array, and the semi-contact width was also wider than that of the roller above the gap between dimple arrays. The average friction was recorded continuously, and a complete cycle of high-speed data was recorded at a sampling rate of $20 \mathrm{kHz}$ every $2 \mathrm{~min}$. In order to verify the reproducibility of results, each test was repeated twice, except for the baseline tests with smooth surfaces which were repeated three times. 


\section{Results and Discussion}

\subsection{Topography Measurements}

All surfaces were examined using the optical (non-contact) Alicona Infinite-Focus profilometer (Alicona Imagine $\mathrm{GmbH}$, Raaba, Austria) prior to reciprocating testing, high-resolution images and accurate 3D surface profiles (measurements achieve a vertical resolution of up to $10 \mathrm{~nm}$ ) were obtained to examine sample finish. For each specimen, five dimples were randomly picked and measured to check the surface topography of the dimples. As shown by an example of sample TF3 in Fig. 2, the interval length was taken between triangles' vertexes, side length ' $a$ ' and height length ' $b$ ' measurements are shown with $L 1$ and $L 2$, respectively, while the 3 -D profiles of each sample design are presented in Fig. 3. From Fig. 2, it can be seen that round corners are achieved instead of designed sharp vertexes, which is due to the fact that for laser structuring a focus diameter of about $20 \mu \mathrm{m}$ was applied defining the minimum achievable curvature radius of a surface pattern. As can be seen from Fig. 3, the triangular sloped dimples are accurately finished, although individual layer steps can be observed in the sloped bottoms $\left(R_{\mathrm{a}} \approx 0.5 \mu \mathrm{m}\right)$, which is due to the intrinsic nature of the layer-by-layer

Fig. 2 Triangular sloped (TS) dimple topography measurements
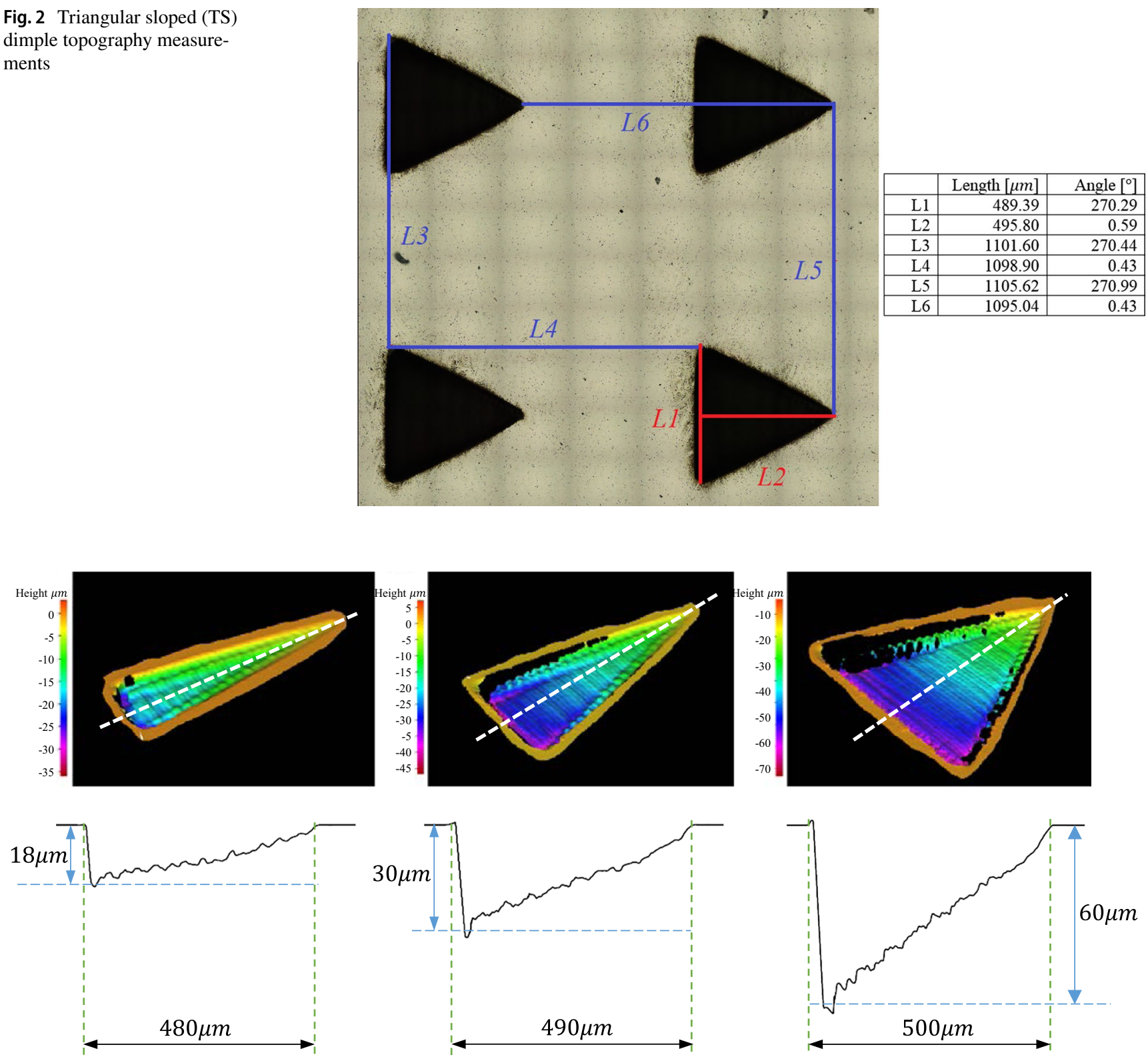

Fig. 3 Triangular sloped (TS) dimple topography with cross-sectional profiles 
laser ablation technique. According to the measurements of all dimple in Table 2, the larger and deeper samples are finished more accurately and the laser texturing technique had a comparably better control with dimple top shape than the cross-sectional shape. In the following analysis, the actual measured parameters will be used rather than the originally designed shape parameters.

After the tests, plate specimens and cylinders were examined where the locations of the dimple arrays were identified from the images presented in Fig. 4. The wear scars on the plate specimens were measured using a contact Taylor Hobson Form Talysurf 120L stylus profilometer (Taylor Hobson Ltd., Leicester, UK). According to the profile measurements, the maximum wear depth was only $100 \mathrm{~nm}$ for all specimens with no obvious wear grooves. Moreover, the roughness difference between the tested zone and non-tested zone was less than $0.001 \mu \mathrm{m}$, which suggests only polishing type wear occurred during tests. While for the tested rollers, all samples have a similar wear scar width: $100 \mu \mathrm{m}$ due to the 'cheese grater effect' on the cylinders, the wear scars on the positions above dimples were slightly deeper than other parts. The specific wear rates for the plate samples were around $18.8 \times 10^{-19} \mathrm{~m}^{3} / \mathrm{Nm}$, and the value for cylinders is less than $4.2 \times 10^{-19} \mathrm{~m}^{3} / \mathrm{Nm}$. The wear volume of both textured plates and cylinders were found to be similar with the non-textured samples.

\subsection{Friction Results}

Before discussing the test results, the stability of the friction results was assessed. The relative standard error of the mean mid-stroke friction coefficient between two tests was found to be about $3 \%$ for the smooth (untextured) samples, and less than $5 \%$ for textured sample results, confirming that the testing process is stable and within expected experimental repeatability. There is negligible variation for both friction and roller displacements over time for all specimens. Also, high frequency and low amplitude friction fluctuations were observed in all friction recordings due to unavoidable noise.

The Stribeck curve of the mid-stroke data of the triangular sloped bottom dimples is presented in Fig. 5. The non-textured results are adapted from a paper published by the current authors [33]. As shown in Fig. 5, at the starting point of the test (hydrodynamic part), the differences in the friction coefficient between the converging and diverging directions are very small for all three surfaces. As the sliding frequency decreases, the directional friction begins to rise around $10 \mathrm{~Hz}$ and below. The friction difference between opposite sliding directions decreases slowly for a few test conditions in the mixed lubrication regime $(350 \mathrm{~N}$, $15-10 \mathrm{~Hz}$ ), and then starts to increase again until the end of the test in boundary lubrication regime, and the biggest friction difference can be observed at the very end of the
Table 2 Measurements of triangular sloped (TS) bottom dimple samples' wear scar

\begin{tabular}{llll}
\hline Sample no. & TS1 & TS2 & TS3 \\
\hline Total dimple arrays in wear scar & 18 & 13 & 9 \\
Total dimples in wear scar & Around 288 & Around 143 & Around 72 \\
Wear scar length (horizontal direction) $(\mu \mathrm{m})$ & 10,185 & 10,188 & 10,184 \\
Wear scar width (vertical direction) $(\mu \mathrm{m})$ & 9160 & 9215 & 9201 \\
First dimple left edge positions $(\mathrm{mm})$ & -4.69 & -4.739 & -4.86 \\
First dimple right edge positions $(\mathrm{mm})$ & -4.21 & -4.249 & -4.36 \\
\hline
\end{tabular}

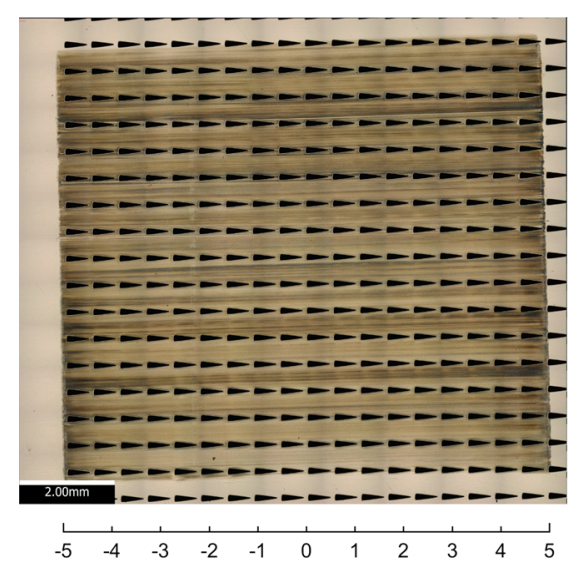

(a)

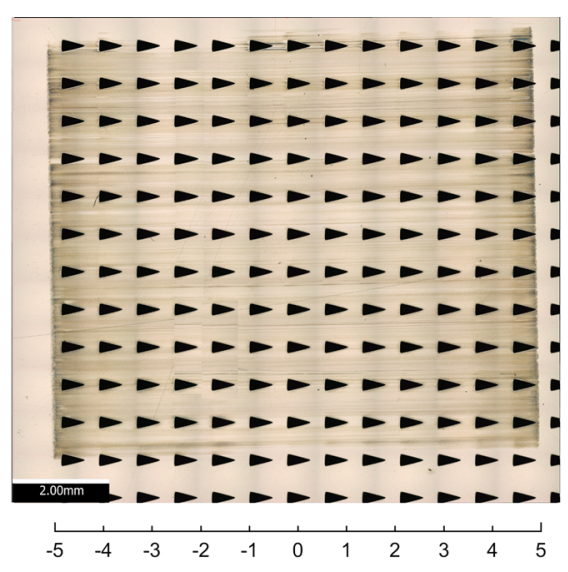

(b)

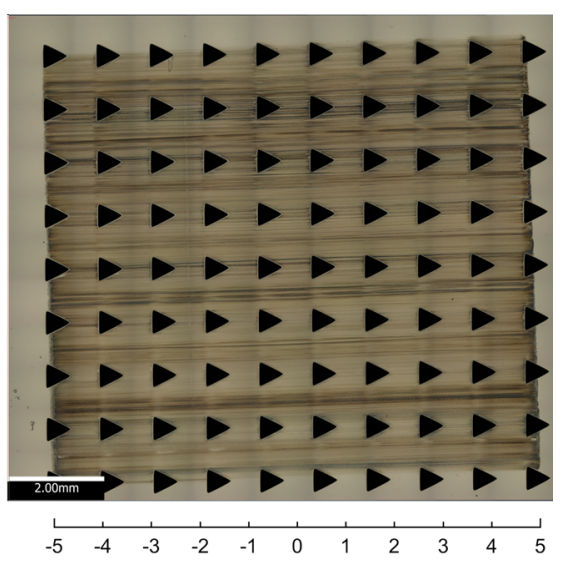

(c)

Fig. 4 Triangular sloped (TS) bottom dimple samples' wear scar topography a TS1, b TS2, c TS3 
Fig. 5 Stribeck curves for TS1, TS2 and TS3 samples

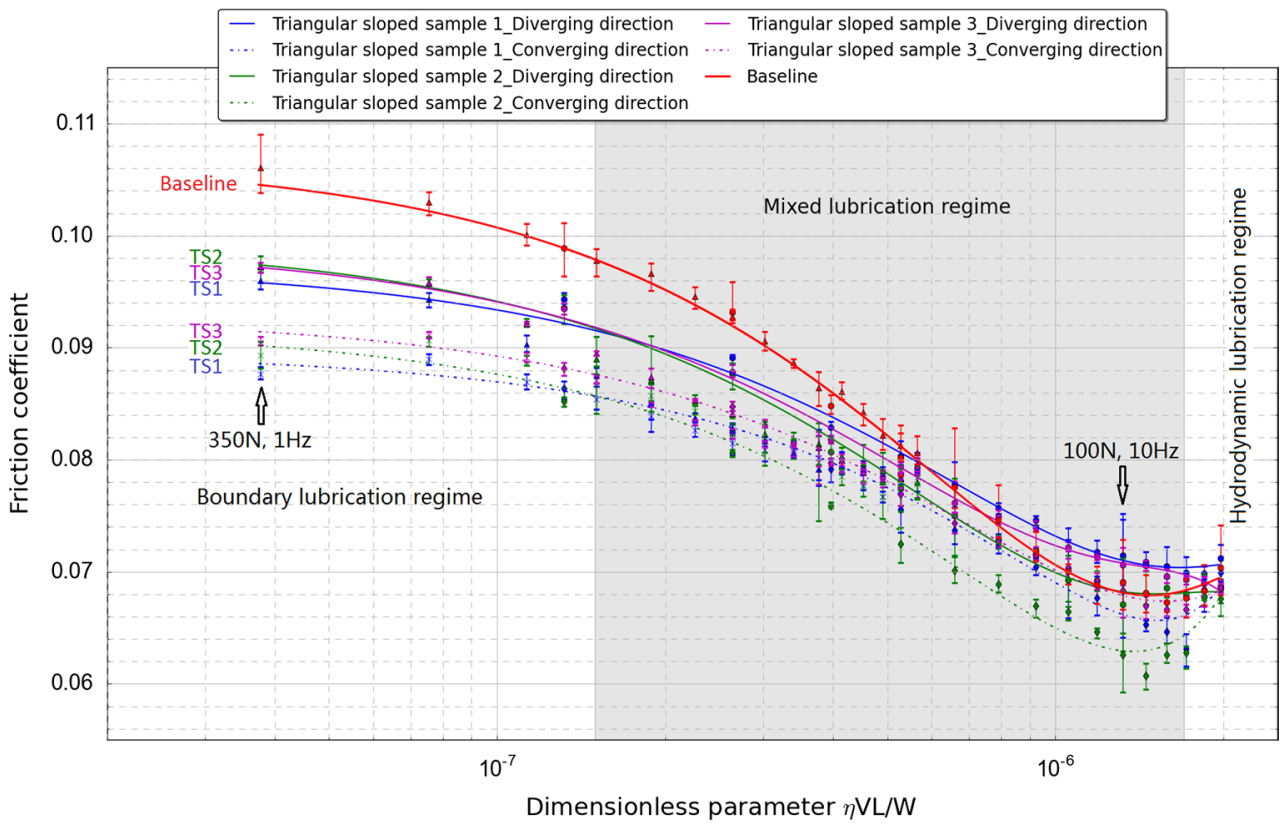

whole test under the test condition $350 \mathrm{~N}, 1 \mathrm{~Hz}$ with a difference of $8-9 \%$.

In general, under mixed to hydrodynamic lubrication conditions, the load is carried partly by contacting asperities and partly by an EHL fluid film, $P=P_{\mathrm{a}}+P_{\mathrm{l}}$, where $P_{\mathrm{a}}$ represents the load carried by solid asperities and $P_{1}$ is the load carried by the fluid film. The friction also consists of two parts, $F=F_{\mathrm{a}}+F_{\mathrm{l}}$, where $F_{\mathrm{a}}$ is the friction caused by the asperity contact and $F_{1}$ is the friction from shearing stress in the lubricant film. Since the normal load applied on the roller is a constant value under each condition, the more load the lubricant is carrying, the less load would be applied on the contact asperities, and the total friction is reduced accordingly. As can be seen from the Stribeck curve, the friction reduction effect of dimples on friction in the mixed to the hydrodynamic regime is minimal. Moreover, some samples are even presenting a high friction than that of the smooth samples, which suggests that, under the mixed to hydrodynamic lubrication regimes, the lubricant film was interrupted by the dimples and that the lubricant film was thinner than that of the smooth surface, leading to more asperities in contact. However, as the converging direction friction is always smaller than that of the diverging direction through all test conditions, that a bigger load was supported by the lubricant when the roller was sliding in converging direction. While the fact that samples with dimples in different size show a similar friction coefficient trend may be because the Hertzian contact width between the roller and plate was much smaller than the dimple sizes. Therefore, no single dimple array was fully covered by the roller, which makes it difficult to generate hydrodynamic lift. However, it should be noticed that, due to the large ratio of dimple size to Hertzian contact width, the hydrodynamic lift is not obvious with these dimples. The lubrication enhancement still exists in other lubrication regimes proves the dimples could reduce friction via other mechanisms, such as: (1) the reduction of contact areas [36], (2) inlet suction as the cavitation zone within the pocket draws more lubricant between contact surfaces [37], and (3) secondary lubrication as oil is drawn out of dimples [38]. In summary, the size of the dimples has little influence on the friction coefficient, and no consistent trend can be found with the dimple size. In particular, in the mixed to hydrodynamic lubrication regimes, the dimples' effect on carrying load was not significant because it is difficult to generate hydrodynamic lift when the contact width is much smaller than the dimple size. But it doesn't indicating the converging angle's influence from both plane can be neglected when dimples are fully covered by the contact area.

In summary, the deformations of contact surfaces could help to squeeze the lubricant out of dimples due to the Tripp effect. However, the moving roller could entrain the lubricant attached on its surfaces into the contact. Those phenomena occur when a thin lubricant film exits between friction pairs, and the solid surfaces are partly in contact.

Apart from the middle stroke friction variation, both friction variation along the whole stroke length and local friction response of dimple arrays were also investigated. According to the high-speed data recordings, no obvious local dimple response can be distinguished under high frequency test conditions, while under the high load and low sliding frequency conditions, both directional friction properties and local friction response can be observed. The 
detailed analysis on how the dimple array's effects the friction under different lubrication regimes is discussed below.

Figure 6 presents the friction response of samples under the test condition $350 \mathrm{~N}$ and $2 \mathrm{~Hz}$, the dashed lines show the friction of untextured sample for comparison. As can be seen from this figure, both the textured and untextured sample has a friction spike at the beginning of the stroke, which is caused by the collapse of squeeze film (the behaviour has been observed previously by Vldescu [17]). Also, the directional friction properties are quite obvious with these anisotropically textured dimples, where the diverging direction friction is always higher than that of the converging direction through the whole stroke. Comparably, the friction difference at the beginning of strokes is much bigger than that of ending point of strokes, which suggests the converging shape of dimples has a bigger impact in the speed increasing period. However, by comparing the friction trends of textured and untextured samples, it can be observed that the friction reduction effects at the strokes' ending points are significant, which suggests the local variation of friction was accumulated together, and the accumulation effect was more obvious with the triangular sloped bottom dimples than other textured samples. In addition, the friction fluctuations for samples TS1 in both directions are increasing with the sliding distance, which might suggest that the local friction response could be accumulated, and become more obvious when each dimple array is close to the next one. For these anisotropic textured surfaces, the friction in converging direction is lower than that of the diverging directions under all test conditions. These outcomes agree with the theoretical analysis made by Nanbu [35], that a thicker lubrication film is easier to be generated with micro-step and micro-wedge bottom shapes, therefore a smaller coefficient of friction is expected in boundary to mixed lubrication regimes. Also, the directional friction properties are in agreement with the experimental observations by Wang [39] and Xie [40].

Moreover, if we compare the results with the other shapes previously studied in this project, see Fig. 7. It should be mentioned that the other three types of dimples in paper [32] have the same dimple side length with the current triangular sloped shape, although the TS3 shaped dimples are four times the depth of the other type dimples.

From the results comparison, the frictions of square flat bottom dimples are almost the same in both directions, while continuous friction difference appeared with the anisotropic textured dimples. As mentioned in the discussion in paper [32], the directional friction properties of square sloped bottom dimples appears at the overall friction trends, and the local frictional response is almost the same with the square flat bottomed dimples. While, the triangular flat dimples have less influence on total friction trends, but cause a larger difference in local friction response. By comparing the triangular sloped dimple with the other two anisotropic shapes, it can be observed that the total friction trend matches to those from square sloped bottom dimples, with the local friction response being more like the triangular at dimples. Moreover, an accumulation effect can be observed with all the dimples with sloped bottoms (square sloped and triangular sloped dimples) in Fig. 7, that the friction difference between opposite directions becomes smaller in the end of stroke. Moreover, for these anisotropic textured dimples, the friction of both converging and diverging directions are in agreement. Also, the friction reduction effects are accumulated by the dimples secondary lubrication, where lubricant inside dimples are easier to be squeezed out, instead of pressure build up by converging shapes.

Before analysing the local friction response of single dimple array in more detail, the real contact length between contact specimens should be clarified. As shown by the real contact length (red line) in Fig. 8a, when the roller's position is just above the dimple array, the actual contact length should be the length of the roller with sum of the dimples length subtracted. The actual contact length varies with the roller's motion, and the fluctuation of the real contact length and the friction with trend line subtracted could be plotted against roller displacement, see Fig. 8b, which adopted from paper [32].

The local friction responses are characterised by comparing them with other shaped dimples under the boundary lubrication regime $(350 \mathrm{~N}, 2 \mathrm{~Hz})$. The friction fluctuations of all four shapes are plotted against the roller displacements using the same data set with Fig. 7, but with trend lines subtracted, see Fig. 9.

For the square flat dimples (SF) results shown in Fig. 9a, friction force decreases abruptly from $F_{0}$ to $F_{1}$ as soon as a pocket array enters the contact. After that, the friction rises slowly within the dimple to $F_{2}$ and reaches the peak value $F_{3}$ at the trailing edge of the dimple array. Finally, it falls back to $F_{4}$, which is a similar value to the initial friction $F_{0}$. The converging and diverging direction friction of the square sloped textured sample (SS) are shown in Fig. 9b, c; the local friction responses are similar when the roller is sliding in the opposite direction, and no obvious friction variation changes can be observed when changing the dimple's bottom shape. Also, the converging sloped at the trailing edge did not help to reduce the friction increase, while for the triangular dimples, the local friction responses of opposite sliding directions are totally different. As shown in Fig. 9d, e, f, g, when the roller is sliding towards the converging direction, the friction decreases rapidly when the dimple array enters the contact. After that, the slow rise within dimple array is skipped over and it rose directly to the peak, $F_{3}$. Finally, it falls back to the average value, $F_{4}$. When the roller is sliding towards the diverging direction, the friction variation when the dimple array enters the contact zone is minimum. 
Fig. 6 Friction force measurements versus time of triangular sloped dimples a TS1, b TS2, c TS3

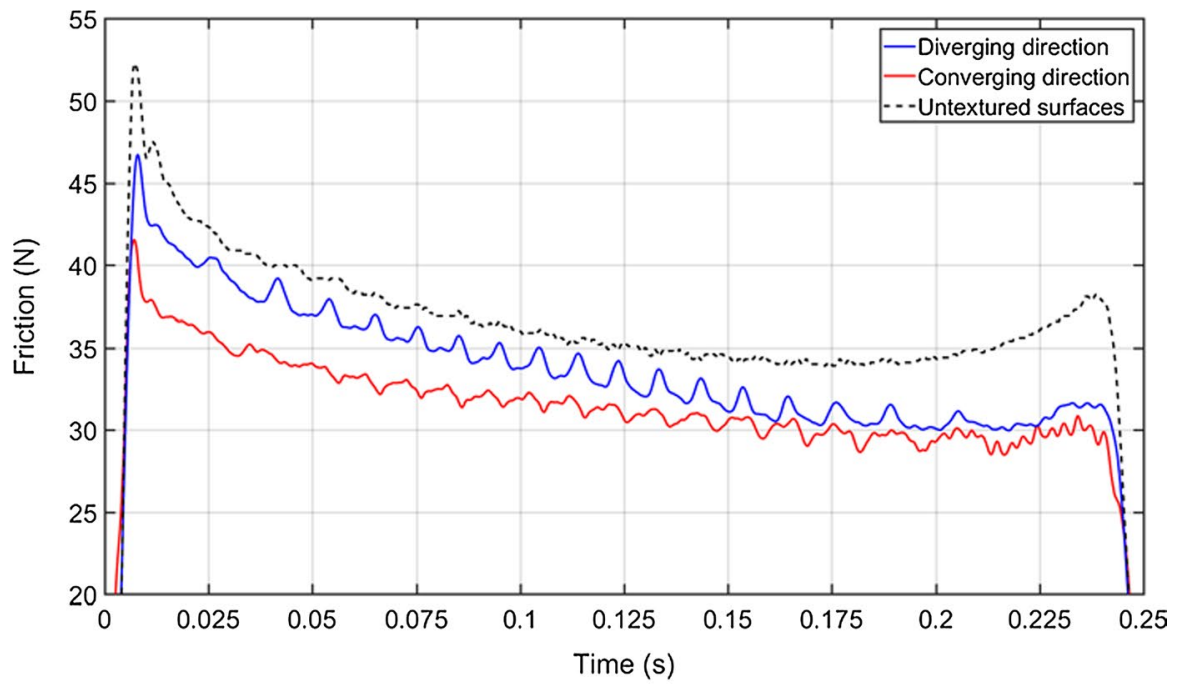

(a)

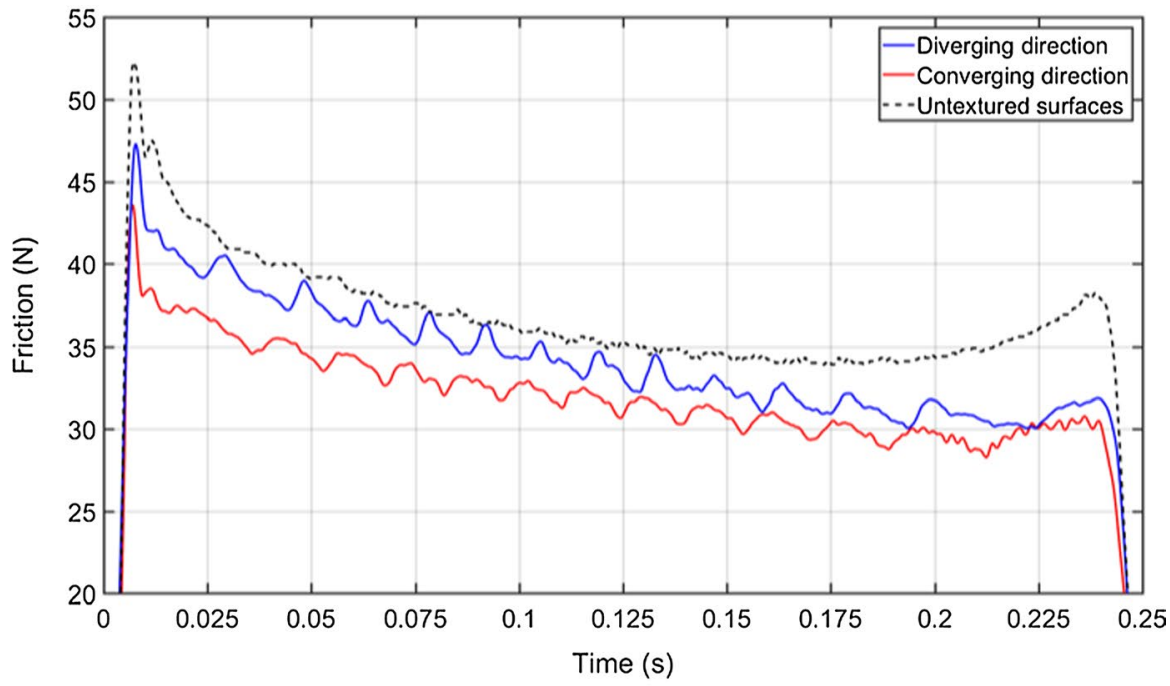

(b)

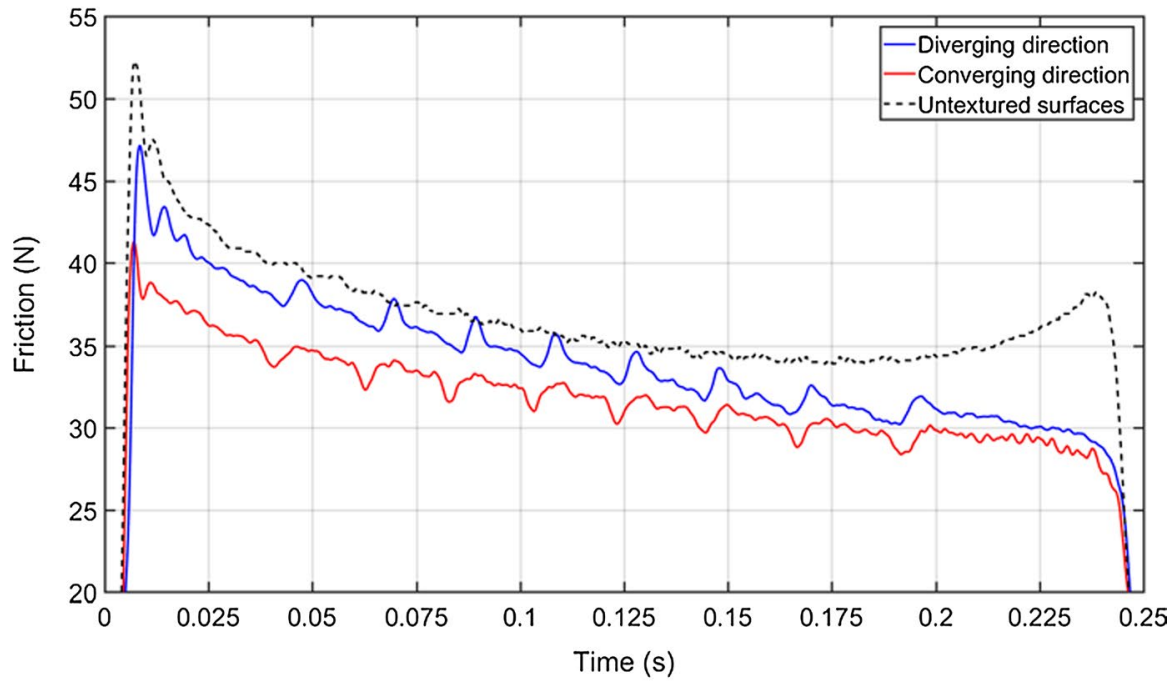

(c) 

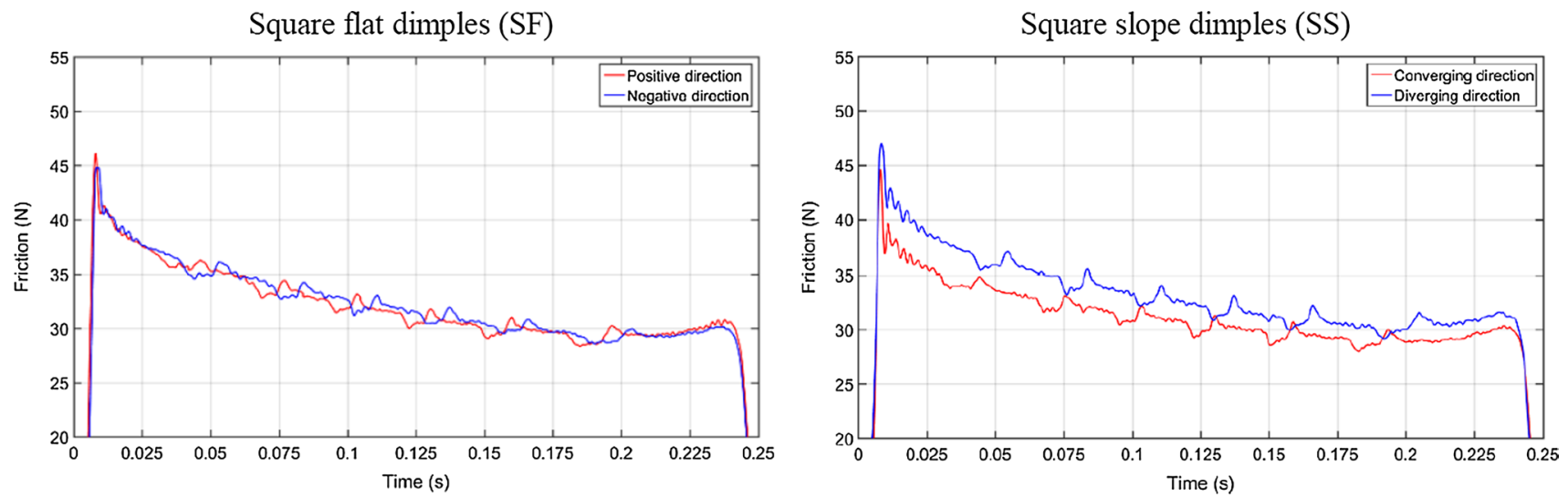

Triangular flat dimples (TF)
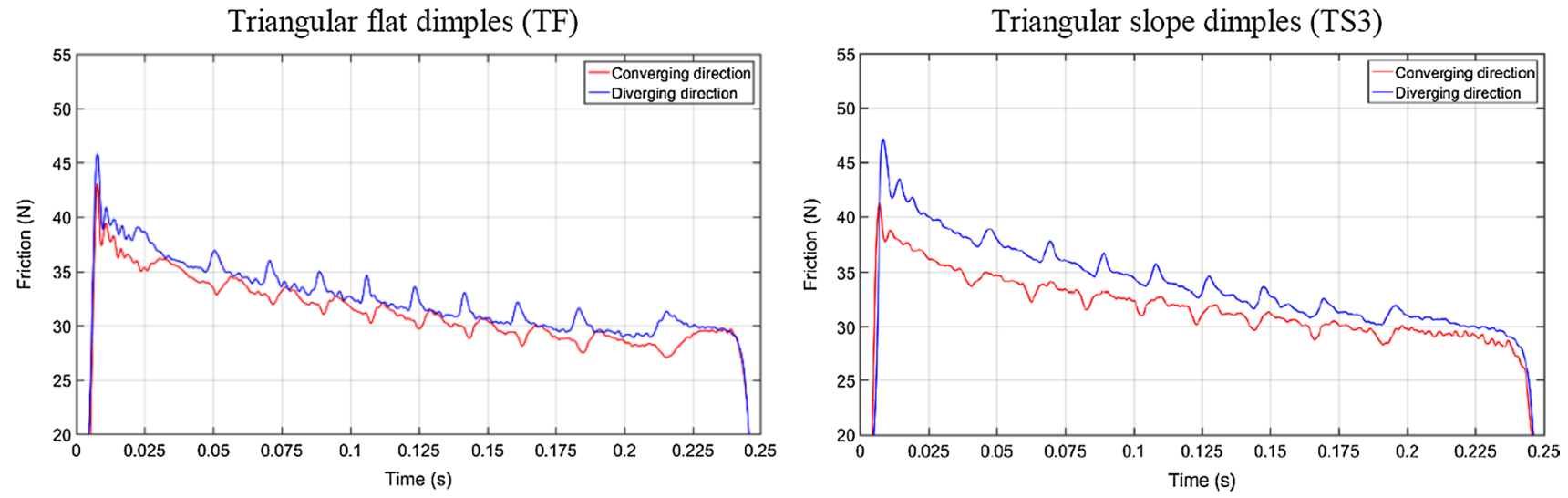

Fig. 7 Friction force measurements versus time of all design shapes, partly adopted from [32]

After that, a sharp increase can be observed at the trailing edge of the triangular dimples, and it falls back abruptly to $F_{4}$. Compared to the triangular flat dimples' results, the friction fluctuation amplitude for triangular sloped bottom dimples did not increase with the assistance of the sloped bottoms. Returning to the local friction and making comparisons between square flat bottom dimples and the square sloped bottom dimples, a similar conclusion can be generated that the sloped bottom could not influence the local friction response of dimples when the dimple size was much larger than the contact width.

From the above comparison and discussion, it could be summarised that, at the boundary of dimple arrays, the friction suddenly drops with the real contact length when the roller is sliding towards the converging direction. While in the opposite sliding direction, the friction peak occurs as soon as the roller meeting the trailing edge of triangular dimples, which has a sudden increase in the real contact length. The dimple arrays bring friction peaks in the diverging direction, while they introduce friction troughs in the converging direction. It may also be caused by the cavitation phenomenon. According to Vladescu et al. [16, 21], the cavitation zone is highly dependent on the top-view of dimple shapes.
In brief, transient friction data captured the local friction response of textures successfully, and the results have shown that the converging shape in the top-view $x-y$ plane (triangular shape dimples) has a greater local response than the transverse $y-z$ plane profile (sloped bottom) when the feature is not capped in the contact area. Although the local friction effect of sloped bottom shapes is not obvious, the total friction reduction has proved that the converging bottom has a beneficial effect in lubrication enhancement. Steps in the leading edge of dimples are beneficial for friction reduction irrespective of dimple geometry. Further experiments with optical transparent materials could be conducted to verify whether cavitation phenomenon enhanced the directional effect.

In this paper, the results of all anisotropic textured surfaces were presented. The Stribeck curves of repeated tests show that the relative standard error of the mean mid-stroke friction coefficient between tests was less than 5\%, which confirms that the test results are reproducible. The friction reduction effect of ultrafast laser textured surfaces was compared with non-textured samples and shows that the dimples on the surface could bring beneficial friction reduction effect, especially in boundary lubrication regimes. Also, the directional friction effect generated by the anisotropic 
Fig. 8 a Real contact length between roller and surface when roller sliding above a dimple array. b Real contact length versus roller position, adopted from [32]

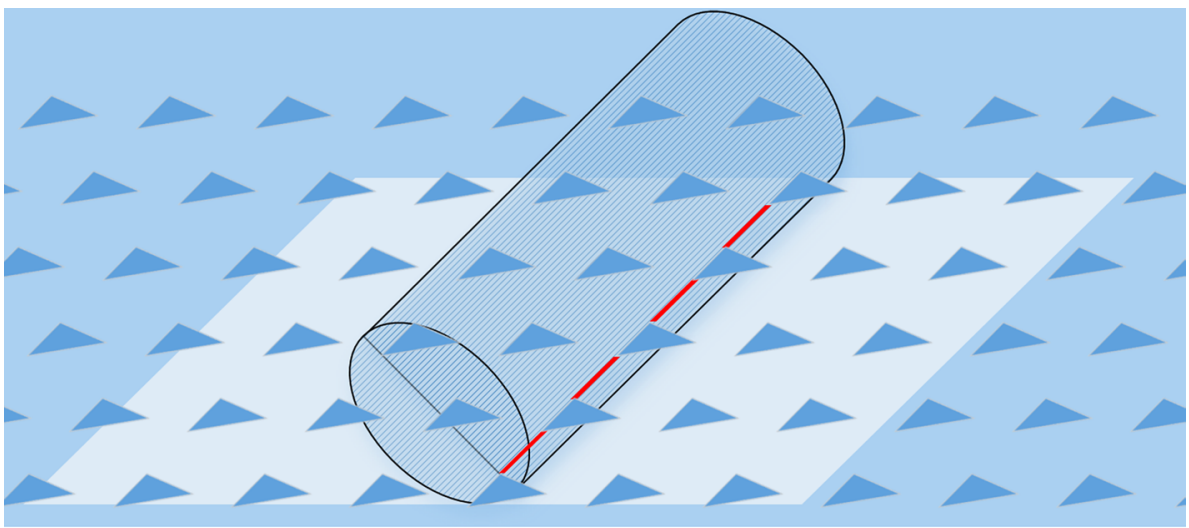

(a)

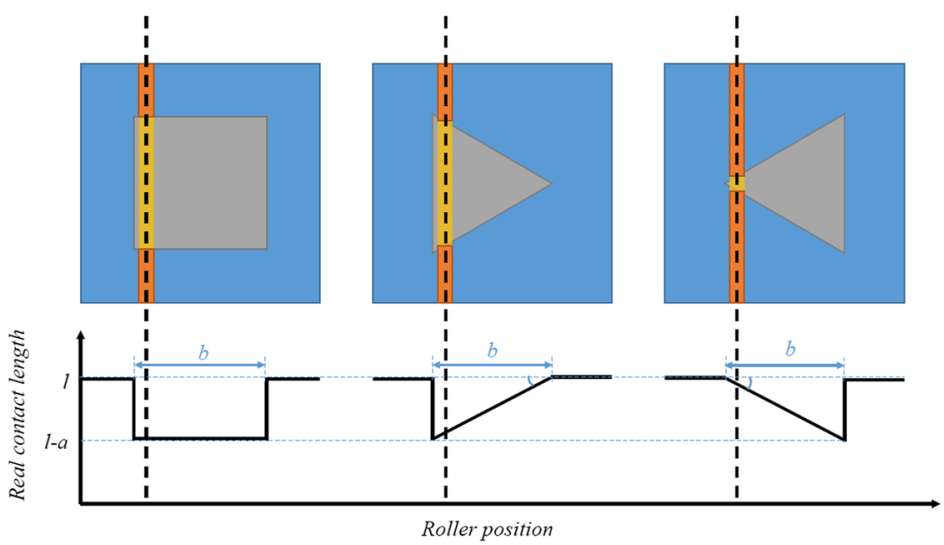

(b) dimpled surfaces suggests the converging/diverging shape in the transverse $y-z$ plane and top-view $x-y$ plane could both influence the frictional properties of the surface. As illustrated by the transient friction response of triangular sloped bottom dimples under boundary lubrication regimes, the converging slope at the trailing edge did not help to reduce the friction peak, which suggests the hydrodynamic lift effect generated by the converging slope is not as significant as the influence of the real contact length.

\section{Conclusions}

The assessment of surface metrology of textured surfaces has shown the possibility of using ultrafast laser surface texturing techniques for the flexible fabrication of complicated texture structures. However, their dimensional accuracy should be further improved by using shorter focus diameter of laser for a more precise contour structuring, and by applying subsequent laser polishing for improving the surface roughness in the cavities, so that the geometry effect of the dimpled surface with exactly the same parameters can be better compared.
- The transient friction behaviour under the boundary lubrication regime of triangular flat bottomed and sloped micro-dimples indicates the real contact length between roller and plate is crucial. The friction drops with the real contact length, and the friction peak occurs with the sudden increase in the real contact length.

- Clearly directional friction effects are produced (maximum 8-9\% friction difference in mid-stroke under the test condition $1 \mathrm{~Hz}, 350 \mathrm{~N}$ ). The transverse $y-z$ plane and the top-view $x-y$ plane both influence the frictional properties of the surface, which attributed to the variation of real contact length and lubrication enhancement such as fluid pressure fluctuations caused by cavitation and secondary lubrication effects.

- Comparing to the rectangular sloped dimple, the friction reduction effects of triangular dimples are better accumulated at the end of each stroke. In addition, triangular sloped bottom dimples has a larger overall directional friction difference along the whole stroke compared to the triangular flat bottom one.

- Due to the large ratio of dimple size to Hertzian contact width, the size influence is minimum. 


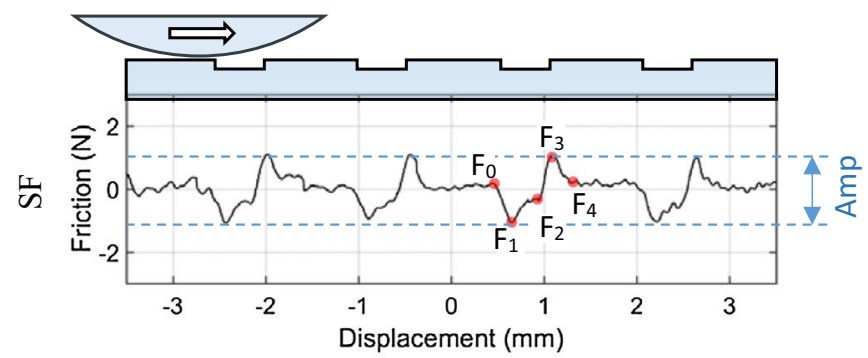

(a)

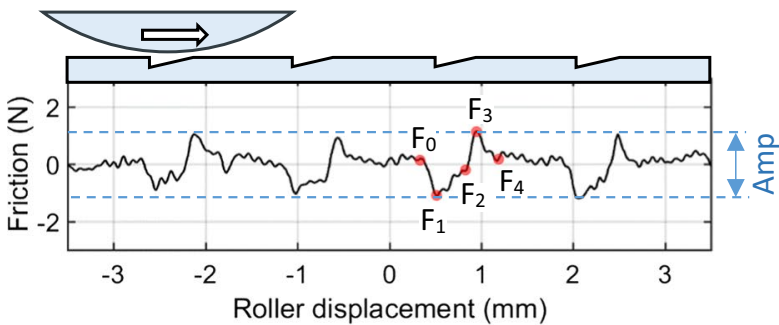

(b)

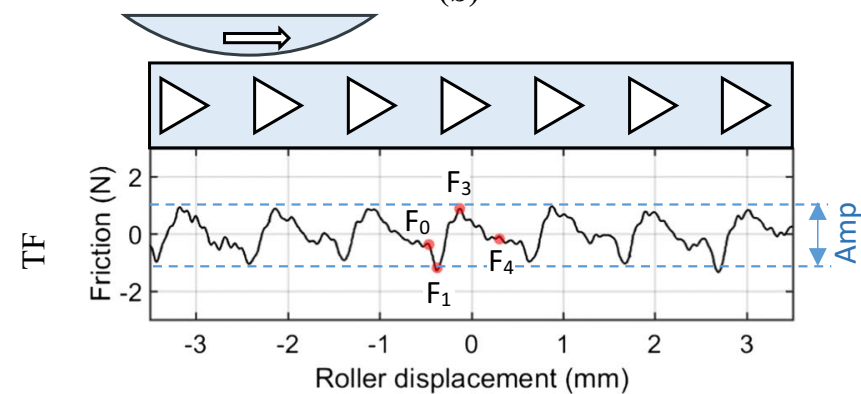

(d)

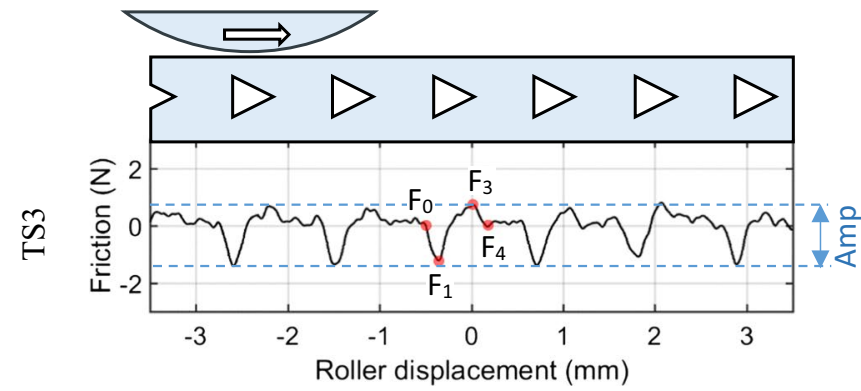

(f)

Fig. 9 Local friction response of textured samples with trend line subtracted under test condition $350 \mathrm{~N}$ and $2 \mathrm{~Hz}$ a square flat (SF) forward direction, b square sloped (SS) converging direction, $\mathbf{c}$ square sloped (SS) diverging direction, d triangular flat (TF) converging

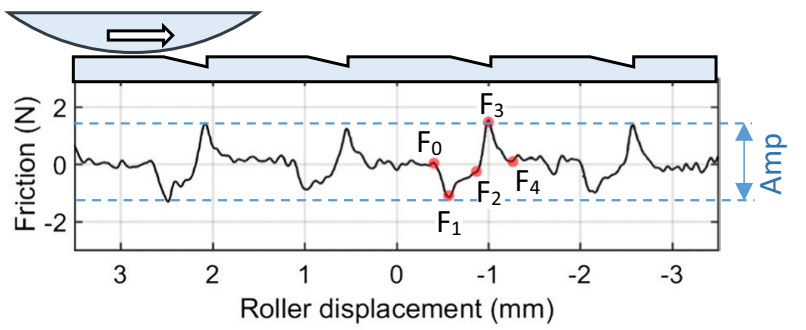

(c)

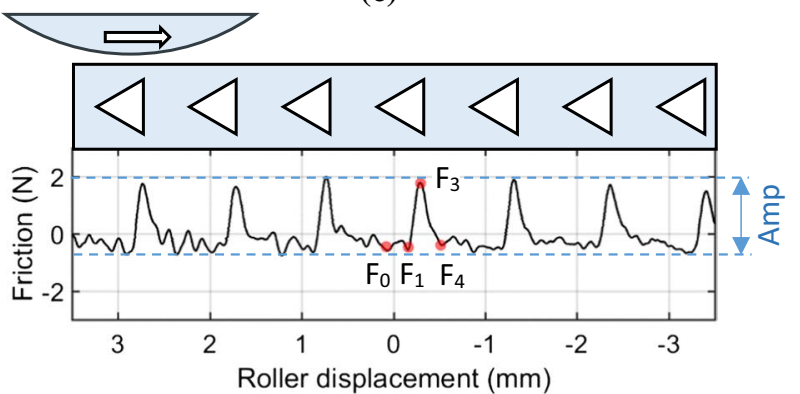

(e)

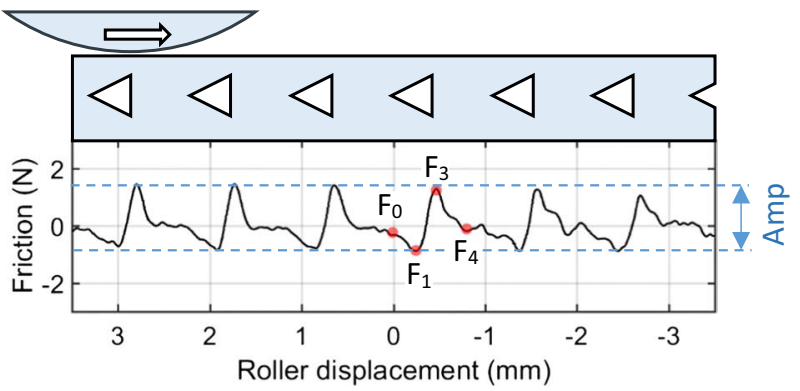

(g)

direction, e triangular flat (TF) diverging direction, $\mathbf{f}$ triangular sloped (TS) converging direction, g triangular sloped (TS) diverging direction

Open Access This article is distributed under the terms of the Creative Commons Attribution 4.0 International License (http://creativeco mmons.org/licenses/by/4.0/), which permits unrestricted use, distribution, and reproduction in any medium, provided you give appropriate credit to the original author(s) and the source, provide a link to the Creative Commons license, and indicate if changes were made.
Acknowledgements This work was sponsored in part by the National Physical Laboratory in the UK. The authors gratefully acknowledge extremely valuable scientific discussion with our colleagues from nCATS. The laser structuring work has received funding from the European Union's Horizon 2020 research and innovation programme under the Marie Sklodowska-Curie Grant Agreement No. 644971. Finally, the support for laser processing by the Karlsruhe Nano Micro Facility (http://www.knmf.kit.edu/) is gratefully acknowledged. 


\section{References}

1. Martin, S., Bhushan, B.: Modeling and optimization of sharkinspired riblet geometries for low drag applications. J. Colloid Interface Sci. 474, 206-215 (2016). https://doi.org/10.1016/j. jcis.2016.04.019

2. Han, Z., Mu, Z., Yin, W., Li, W., Niu, S., Zhang, J., Ren, L.: Biomimetic multifunctional surfaces inspired from animals. Adv. Colloid Interface Sci. 234, 27-50 (2016). https://doi. org/10.1016/j.cis.2016.03.004

3. Koch, K., Bhushan, B., Barthlott, W.: Diversity of structure, morphology, and wetting of plant surfaces. Soft Matter. 4, 1943-1963 (2008)

4. Bhushan, B., Jung, Y.C.: Natural and biomimetic artificial surfaces for superhydrophobicity, self-cleaning, low adhesion, and drag reduction. Prog. Mater. Sci. 56(1), 1-108 (2011). https:// doi.org/10.1016/j.pmatsci.2010.04.003

5. Gao, H., Wang, X., Yao, H., Gorb, S., Arzt, E.: Mechanics of hierarchical adhesion structures of geckos. Mech. Mater. 37(2), 275-285 (2005). https://doi.org/10.1016/j.mechm at.2004.03.008

6. Gropper, D., Wang, L., Harvey, T.J.: Hydrodynamic lubrication of textured surfaces: a review of modeling techniques and key findings. Tribol. Int. 94, 509-529 (2016)

7. Kango, S., Sharma, R., Pandey, R.: Thermal analysis of microtextured journal bearing using non-Newtonian rheology of lubricant and JFO boundary conditions. Tribol. Int. 69, 19-29 (2014)

8. Antoszewski, B.: Mechanical seals with sliding surface texture-model fluid flow and some aspects of the laser forming of the texture. Proc. Eng. 39, 51-62 (2012)

9. Marian, V.G., Gabriel, D., Knoll, G., Filippone, S.: Theoretical and experimental analysis of a laser textured thrust bearing. Tribol. Lett. 44(3), 335 (2011)

10. Zhou, Y., Zhu, H., Tang, W., Ma, C., Zhang, W.: Development of the theoretical model for the optimal design of surface texturing on cylinder liner. Tribol. Int. 52, 1-6 (2012)

11. Kovalchenko, A., Ajayi, O., Erdemir, A., Fenske, G., Etsion, I.: The effect of laser texturing of steel surfaces and speed-load parameters on the transition of lubrication regime from boundary to hydrodynamic. Tribol. Trans. 47(2), 299-307 (2004)

12. Podgornik, B., Vilhena, L., Sedlaček, M., Rek, Z., Žun, I.: Effectiveness and design of surface texturing for different lubrication regimes. Meccanica 47(7), 1613-1622 (2012)

13. Scaraggi, M., Mezzapesa, F.P., Carbone, G., Ancona, A., Tricarico, L.: Friction properties of lubricated laser-microtexturedsurfaces: an experimental study from boundary-to hydrodynamic-lubrication. Tribol. Lett. 49(1), 117-125 (2013)

14. Braun, D., Greiner, C., Schneider, J., Gumbsch, P.: Efficiency of laser surface texturing in the reduction of friction under mixed lubrication. Tribol. Int. 77, 142-147 (2014)

15. Scaraggi, M., Mezzapesa, F.P., Carbone, G., Ancona, A., Sorgente, D., Lugarà, P.M.: Minimize friction of lubricated lasermicrotextured-surfaces by tuning microholes depth. Tribol. Int. 75, 123-127 (2014)

16. Profito, F.J., Vlădescu, S.-C., Reddyhoff, T., Dini, D.: Transient experimental and modelling studies of laser-textured micro-grooved surfaces with a focus on piston-ring cylinder liner contacts. Tribol. Int. 113, 125-136 (2017). https://doi. org/10.1016/j.triboint.2016.12.003

17. Vlădescu, S.-C., Medina, S., Olver, A.V., Pegg, I.G., Reddyhoff, T.: Lubricant film thickness and friction force measurements in a laser surface textured reciprocating line contact simulating the piston ring-liner pairing. Tribol. Int. 98, 317-329 (2016)
18. Ronen, A., Etsion, I., Kligerman, Y.: Friction-reducing surfacetexturing in reciprocating automotive components. Tribol. Trans. 44(3), 359-366 (2001)

19. Ryk, G., Kligerman, Y., Etsion, I.: Experimental investigation of laser surface texturing for reciprocating automotive components. Tribol. Trans. 45(4), 444-449 (2002)

20. Costa, H., Hutchings, I.: Hydrodynamic lubrication of textured steel surfaces under reciprocating sliding conditions. Tribol. Int. 40(8), 1227-1238 (2007)

21. Vladescu, S.-C., Olver, A.V., Pegg, I.G., Reddyhoff, T.: The effects of surface texture in reciprocating contacts: an experimental study. Tribol. Int. 82, 28-42 (2015)

22. Vlădescu, S.-C., Medina, S., Olver, A.V., Pegg, I.G., Reddyhoff, T.: The transient friction response of a laser-textured, reciprocating contact to the entrainment of individual pockets. Tribol. Lett. 62(2), 19 (2016)

23. Vlădescu, S.-C., Olver, A.V., Pegg, I.G., Reddyhoff, T.: Combined friction and wear reduction in a reciprocating contact through laser surface texturing. Wear 358, 51-61 (2016)

24. Morris, N., Leighton, M., De la Cruz, M., Rahmani, R., Rahnejat, H., Howell-Smith, S.: Combined numerical and experimental investigation of the micro-hydrodynamics of chevron-based textured patterns influencing conjunctional friction of sliding contacts. Proc. Inst. Mech. Eng. J J. Eng. Tribol. 229(4), 316$335(2015)$

25. Lu, X., Khonsari, M.: An experimental investigation of dimple effect on the stribeck curve of journal bearings. Tribol. Lett. 27(2), 169 (2007)

26. Etsion, I., Halperin, G., Brizmer, V., Kligerman, Y.: Experimental investigation of laser surface textured parallel thrust bearings. Tribol. Lett. 17(2), 295-300 (2004)

27. Qiu, Y., Khonsari, M.: Experimental investigation of tribological performance of laser textured stainless steel rings. Tribol. Int. 44(5), 635-644 (2011)

28. Henry, Y., Bouyer, J., Fillon, M.: An experimental analysis of the hydrodynamic contribution of textured thrust bearings during steady-state operation: a comparison with the untextured parallel surface configuration. Proc. Inst. Mech. Eng. J J. Eng. Tribol. 229(4), 362-375 (2015)

29. Yu, X., He, S., Cai, R.: Frictional characteristics of mechanical seals with a laser-textured seal face. J. Mater. Process. Technol. 129(1), 463-466 (2002)

30. Etsion, I.: Improving tribological performance of mechanical components by laser surface texturing. Tribol. Lett. 17(4), 733-737 (2004)

31. Bai, L., Bai, S.: Frictional performance of a textured surface with elliptical dimples: geometric and distribution effects. Tribol. Trans. 57(6), 1122-1128 (2014)

32. Lu, P., Wood, R.J.K., Gee, M.G., Wang, L., Pfleging, W.: The use of anisotropic texturing for control of directional friction. Tribol. Int. 113(Supplement C), 169-181 (2017). https://doi. org/10.1016/j.triboint.2017.02.005

33. Lu, P., Wood, R.J., Gee, M.G., Wang, L., Pfleging, W.: The friction reducing effect of square-shaped surface textures under lubricated line-contacts: an experimental study. Lubricants 4(3), $26(2016)$

34. Yu, H., Wang, X., Zhou, F.: Geometric shape effects of surface texture on the generation of hydrodynamic pressure between conformal contacting surfaces. Tribol. Lett. 37, 123-130 (2010)

35. Nanbu, T., Ren, N., Yasuda, Y., Zhu, D., Wang, Q.J.: Microtextures in concentrated conformal-contact lubrication: effects of texture bottom shape and surface relative motion. Tribol. Lett. 29(3), 241-252 (2008)

36. Menezes, P.L., Kailas, S.V.: Role of surface texture and roughness parameters on friction and transfer film formation when 
UHMWPE sliding against steel. Biosurf. Biotribol. 2(1), 1-10 (2016). https://doi.org/10.1016/j.bsbt.2016.02.001

37. Fowell, M., Olver, A.V., Gosman, A.D., Spikes, H.A., Pegg, I.: Entrainment and inlet suction: two mechanisms of hydrodynamic lubrication in textured bearings. J. Tribol. 129, 336-347 (2007)

38. Wang, X., Kato, K.: Improving the anti-seizure ability of SiC seal in water with RIE texturing. Tribol. Lett. 14(4), 275-280 (2003). https://doi.org/10.1023/A:1022650813314
39. Wang, W.-Z., Huang, Z., Shen, D., Kong, L., Li, S.: The effect of triangle-shaped surface textures on the performance of the lubricated point-contacts. J. Tribol. 135(2), 021503 (2013)

40. Xie, Y., Li, Y., Wang, Y., Suo, S., Liu, X.: An experimental investigation of tribological performance of triangular textures in water lubrication regime. Sci. China Phys. Mech. Astron. 57(2), 273-279 (2014) 